\title{
MODELING NEGOTIATION SUBDIALOGUES ${ }^{\mathbf{1}}$
}

\author{
Lynn Lambert and Sandra Carberry \\ Department of Computer and Information Sciences \\ University of Delaware \\ Newark, Delaware 19716, USA \\ email: lambertecis.udel.edu, carberryecis.udel.edu
}

\begin{abstract}
This paper presents a plan-based model that handles negotiation subdialogues by inferring both the communicative actions that people pursue when speaking and the beliefs underlying these actions. We contend that recognizing the complex discourse actions pursued in negotiation subdialogues (e.g., expressing doubt) requires both a multistrength belief model and a process model that combines different knowledge sources in a unified framework. We show how our model identifies the structure of negotiation subdialogues, including recognizing expressions of doubt, implicit acceptance of communicated propositions, and negotiation subdialogues embedded within other negotiation subdialogues.
\end{abstract}

\section{Introduction}

Since negotiation is an integral part of multi-agent activity, a robust natural language understanding system must be able to handle subdialogues in which participants negotiate what has been claimed in order to try to come to some agreement about those claims. To handle such dialogues, the system must be able to recognize when a dialogue participant has initiated a negotiation subdialogue and why the participant began the negotiation (i.e., what beliefs led the participant to start the negotiation). This paper presents a plan-based model of task-oriented interactions that assimilates negotiation subdialogues by inferring both the communicative actions that people pursue when speaking and the beliefs underlying these actions. We will argue that recognizing the complex discourse actions pursued in negotiation subdialogues (e.g., expressing doubt) requires both a multi-strength belief model and a processing strategy that combines different knowledge sources in a unified framework, and we will show how our model incorporates these and recognizes the structure of negotiation subdialogues.

\section{Previous Work}

Several researchers have built argument understanding systems, but none of these has addressed participants coming to an agreement or mutual belief about a particular situation, either because the arguments were only monologues

\footnotetext{
${ }^{1}$ This work is being supported by the National Science Foundation under Grant No. IRI-9122026. The Government has certain rights in this material.
}

(Cohen, 1987; Cohen and Young, 1991), or because they assumed that dialogue participants do not change their minds (Flowers, McGuire and Birnbaum, 1982; Quilici, 1991). Others have examined more cooperative dialogues. Clark and Schaefer (1989) contend that utterances must be grounded, or understood, by both parties, but they do not address conflicts in belief, only lack of understanding. Walker (1991) has shown that evidence is often provided to ensure both understanding and believing an utterance, but she does not address recognizing lack of belief or lack of understanding. Reichman (1981) outlines a model for informal debate, but does not provide a detailed computational mechanism for recognizing the role of each utterance in a debate.

In previous work (Lambert and Carberry, 1991), we described a tripartite plan-based model of dialogue that recognizes and differentiates three different kinds of actions: domain, problemsolving, and discourse. Domain actions relate to performing tasks in a given domain. We are modeling cooperative dialogues in which one agent has a domain goal and is working with another helpful, more expert agent to determine what domain actions to perform in order to accomplish this goal. Many researchers (Allen, 1979; Carberry, 1987; Goodman and Litman, 1992; Pollack, 1990; Sidner, 1985) have shown that recognition of domain plans and goals gives a system the ability to address many difficult problems in understanding. Problem-solving actions relate to how the two dialogue participants are going about building a plan to achieve the planning agent's domain goal. Ramshaw, Litman, and Wilensky (Ramshaw, 1991; Litman and Allen, 1987; Wilensky, 1981) have noted the need for recognizing problem-solving actions. Discourse actions are the communicative actions that people perform in saying something, e.g., asking a question or expressing doubt. Recognition of discourse actions provides expectations for subsequent utterances, and explains the purpose of an utterance and how it should be interpreted.

Our system's knowledge about how to perform actions is contained in a library of discourse, problem-solving, and domain recipes (Pollack, 1990). Although domain recipes are not mutually known by the participants (Pollack, 1990), how to communicate and how to solve problems are com- 
Discourse Recipe-C3: \{_agent1 informs _agent2 of _prop\}

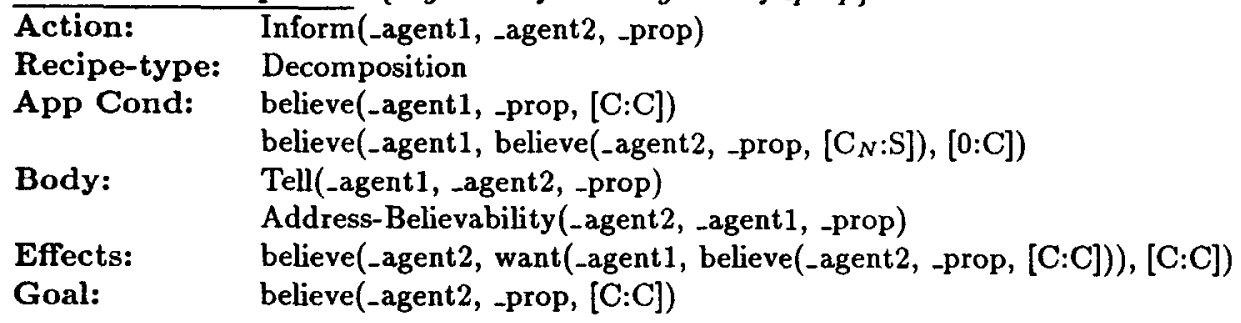

Discourse Recipe-C2:

\{-agent1 expresses doubt to _agent2 about _prop1 because _agent1 believes _prop2 to be true\}

Action: $\quad$ Express-Doubt(_agent1, _agent2, _prop1, _prop2, _rule)

Recipe-type: Decomposition

App Cond: believe(-agent1, -prop2, [W:S])

believe(_agent1, believe(_agent2, _prop1, [S:C]), [S:C])

believe(_agent1, ((-prop2 $\wedge$ _rule) $\Rightarrow \neg$-prop1), [S:C])

believe(-agent1, _rule, $[\mathrm{S}: \mathrm{C}]$ )

in-focus(-prop1))

Body: Convey-Uncertain-Belief(-agent1, _agent2, -prop2)

Address-Q-Acceptance(_agent2, _agent1, _prop2)

Effects: $\quad$ believe(_agent2, believe(_agent1, ,prop1, $\left[\mathrm{S}_{N}: \mathrm{W}_{N}\right]$ ), [S:C])

believe(_agent2, want(_agent1, Resolve-Conflict(_agent2, _agent1, _prop1, _prop2)), [S:C])

Goal:

want(_agent2, Resolve-Conflict(_agent2, _agent1, _prop1, _prop2))

Figure 1. Two Sample Discourse Recipes

mon skills that people use in a wide variety of contexts, so the system can assume that knowledge about discourse and problem-solving recipes is shared knowledge. Figure 1 contains two discourse recipes. Our representation of a recipe includes a header giving the name of the recipe and the action that it accomplishes, preconditions, applicability conditions, constraints, a body, effects, and a goal. Constraints limit the allowable instantiation of variables in each of the components of a recipe (Litman and Allen, 1987). Applicability conditions (Carberry, 1987) represent conditions that must be satisfied in order for the recipe to be reasonable to apply in the given situation and, in the case of many of our discourse recipes, the applicability conditions capture beliefs that the dialogue participants must hold. Especially in the case of discourse recipes, the goals and effects are likely to be different. This allows us to differentiate between illocutionary and perlocutionary effects and to capture the notion that one can, for example, perform an inform act without the hearer adopting the communicated proposition. ${ }^{2}$

As actions are inferred by our process model, a structure of the discourse is built which is referred to as the Dialogue Model, or DM. In the DM, discourse, problem-solving, and domain actions are each modeled on a separate level. Within each of these levels, actions may contribute to other actions in the dialogue, and this is captured with specialization (Kautz and Allen, 1986), sub-

\footnotetext{
${ }^{2}$ Consider, for example, someone saying " $I$ informed you of X but you wouldn't believe me."
}

action, and enablement arcs. Thus, actions at each level form a tree structure in which each node represents an action that a participant is performing and the children of a node represent actions pursued in order to contribute to the parent action. By using a tree structure to model actions at each level and by allowing the tree structures to grow at the root as well as at the leaves, we are able to incrementally recognize discourse, problem-solving, and domain intentions, and can recognize the relationship among several utterances that are all part of the same higher-level discourse act even when that act cannot be recognized from the first utterance alone. Other advantages of our tripartite model are discussed in Lambert and Carberry (1991)

An action on one level in the DM may also contribute to an action on an immediately higher level. For example, discourse actions may be executed in order to obtain the information necessary for performing a problem-solving action and problem-solving actions may be executed in order to construct a domain plan. We capture this with links between actions on adjacent levels of the DM.

Figure 2 gives a DM built by our prototype system whose implementation is currently being expanded to include belief ascription and use of linguistic information. It shows that a question has been asked and answered, that this question/answer pair contributes to the higher-level discourse action of obtaining information about what course Dr. Smith is teaching, that this discourse action enables the problem-solving action of instantiating a parameter in a Learn-Material 


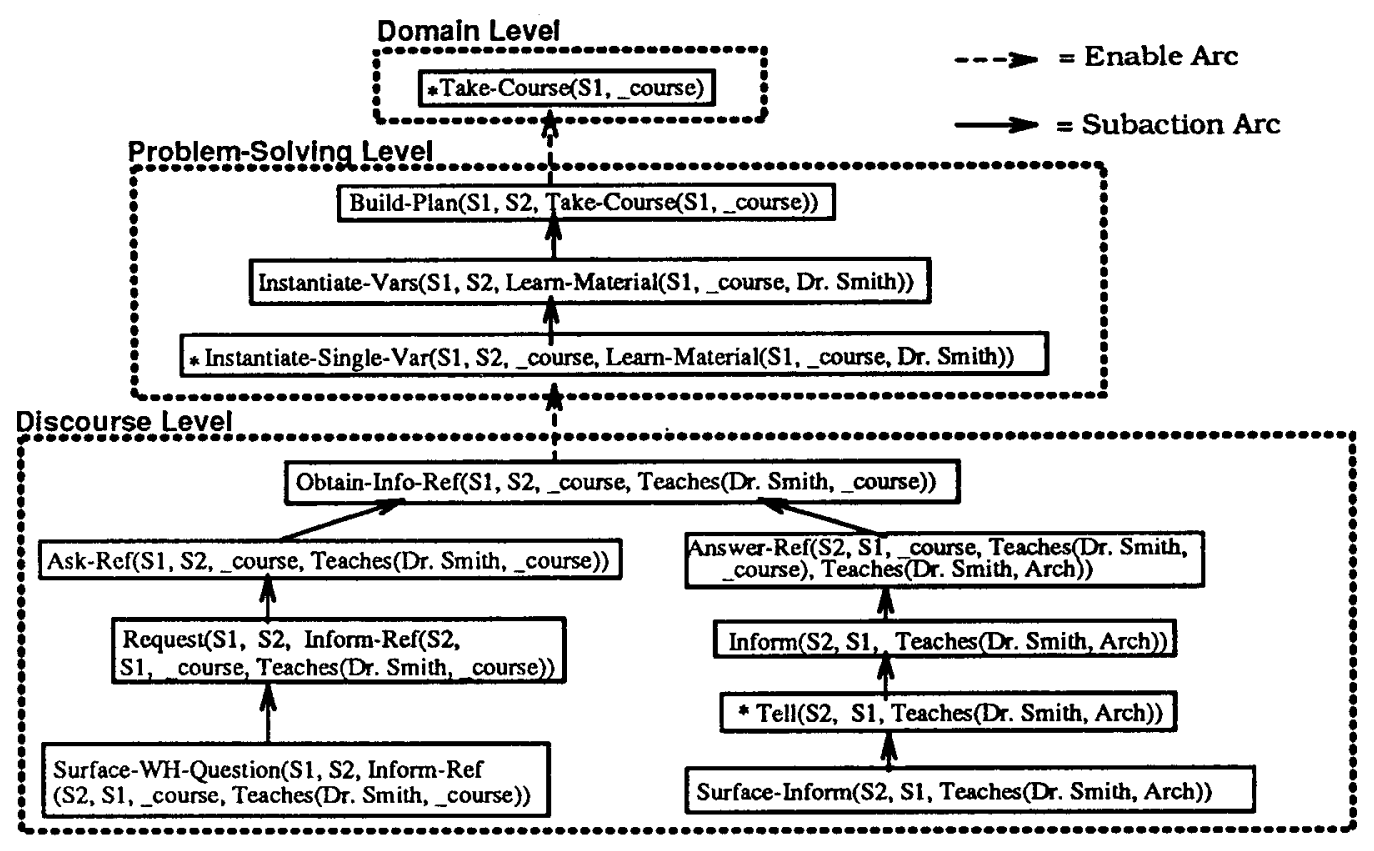

Figure 2. Dialogue Model for two utterances

action, and that this problem-solving action contributes to the problem-solving action of building a plan in order to perform the domain action of taking a course.

The work described in this paper uses our tripartite model, but addresses the recognition of discourse actions and their use in the modeling of negotiation subdialogues.

\section{Discourse Actions and Implicit Acceptance}

One of the most important aspects of assimilating dialogue is the recognition of discourse actions and the role that an utterance plays with respect to the rest of the dialogue. For example, in (3), if S1 believes that each course has a single instructor, then $\mathrm{S} 1$ is expressing doubt at the proposition conveyed in (2). But in another context, (3) might simply be asking for verification.

(1) S1: What is Dr. Smith teaching?

(2) S2: Dr. Smith is teaching Architecture.

(3) S1: Isn't Dr. Brown teaching Architecture?

Unless a natural language system is able to identify the role that an utterance is intended to play in a dialogue, the system will not be able to generate cooperative responses which address the participants' goals.

In addition to recognizing discourse actions, it is also necessary for a cooperative system to recognize a user's changing beliefs as the dialogue progresses. Allen's representation of an Inform speech act (Allen, 1979) assumed that a listener adopted the communicated proposition.
Clearly, listeners do not adopt everything they are told (e.g., (3) indicates that S1 does not immediately accept that Dr. Smith is teaching Architecture). Perrault's persistence model of belief (Perrault, 1990) assumed that a listener adopted the communicated proposition unless the listener had conflicting beliefs. Since Perrault's model assumes that people's beliefs persist, it cannot account for $\mathrm{S} 1$ eventually accepting the proposition that Dr. Smith is teaching Architecture. We show in Section 6 how our model overcomes this limitation.

Our investigation of naturally occurring dialogues indicates that listeners are not passive participants, but instead assimilate each utterance into a dialogue in a multi-step acceptance phase. For statements, ${ }^{3}$ a listener first attempts to understand the utterance because if the utterance is not understood, then nothing else about it can be determined. Second, the listener determines if the utterance is consistent with the listener's beliefs; and finally, the listener determines the appropriateness of the utterance to the current context. Since we are assuming that people are engaged in a ccoperative dialogue, a listener must indicate when the listener does not understand, believe, or consider relevant a particular utterance, addressing understandability first, then believability, then relevance. We model this acceptance process by including acceptance actions in the body of many of our discourse recipes. For example, the actions in the body of an Inform recipe (see Figure 1) are: 1) the speaker (-agent1) tells the listener (_agent2)

\footnotetext{
${ }^{3}$ Questions must also be accepted and assimilated into a dialogue, but we are concentrating on statements here.
} 
the proposition that the speaker wants the listener to believe (-prop); and 2) the listener and speaker address believability by discussing whatever is necessary in order for the listener and speaker to come to an agreement about what the speaker said. ${ }^{4}$ This second action, and the subactions executed as part of performing it, account for subdialogues which address the believability of the proposition communicated in the Inform action. Similar acceptance actions appear in other discourse recipes. The Tell action has a body containing a SurfaceInform action and an Address-Understanding action; the latter enables both participants to ensure that the utterance has been understood.

The combination of the inclusion of acceptance actions in our discourse recipes and the ordered manner in which people address acceptance allows our model to recognize the implicit acceptance of discourse actions. For example, Figure 2 presents the DM derived from utterances (1) and (2), with the current focus of attention on the discourse level, the Tell action, marked with an asterisk. In attempting to assimilate (3) into this DM, the system first tries to interpret (3) as addressing the understanding of (2) (i.e., as part of the Tell action which is the current focus of attention in Figure 2). Since a satisfactory interpretation is not found, the system next tries to relate (3) to the Inform action in Figure 2, trying to interpret (3) as addressing the believability of (2). The system finds that the best interpretation of (3) is that of expressing doubt at (2), thus confirming the hypothesis that (3) is addressing the believability of (2). This recognition of (3) as contributing to the Inform action in Figure 2 indicates that $\mathrm{S} 1$ has implicitly indicated understanding by passing up the opportunity to address understanding in the Tell action that appears in the DM and instead moving to a relevant higher-level discourse action, thus conveying that the Tell action has been successful.

\section{Recognizing Beliefs}

In the dialogue in the preceding section, in order for $\mathrm{S} 1$ to use the proposition communicated in (3) to express doubt at the proposition conveyed in (2), $\mathrm{S} 1$ must believe

(a) that Dr. Brown teaches Architecture;

(b) that S2 believes that Dr. Smith is teaching Architecture; and

(c) that Dr. Brown teaching Architecture is an indication that Dr. Smith does not teach Architecture.

We capture these beliefs in the applicability conditions for an Express-Doubt discourse act (see Figure 1). In order for the system to recognize (3)

4 This is where our model differs from Allen's and Perrault's; we allow the listener to adopt, reject, or negotiate the speaker's claims, which might result in the listener eventually adopting the speakers claims, the listener changing the mind of the speaker, or both agreeing to disagree. as an expression of doubt, it must come to believe that these applicability conditions are satisfied. The system's evidence that S1 believes (a) is provided by S1's utterance, (3). But (3) does not state that Dr. Brown teaches Architecture; instead, $\mathrm{S} 1$ uses a negative yes-no question to ask whether or not Dr. Brown teaches Architecture. The surface form of this utterance indicates that S1 thinks that Dr. Brown teaches Architecture but is not sure of it. Thus, from the surface form of utterance (3), a listener can attribute to $\mathrm{S} 1$ an uncertain belief in the proposition that Dr. Brown teaches Architecture.

This recognition of uncertain beliefs is an important part of recognizing complex discourse actions such as expressing doubt. If the system were limited to recognizing only lack of belief and belief, then yes-no questions would have to be interpreted as conveying lack of belief about the queried proposition, since a question in a cooperative consultation setting would not be felicitous if the speaker already knew the answer. Thus it would be impossible to attribute (a) to S1 from a question such as (3). And without this belief attribution, it would not be possible to recognize expressions of doubt. Furthermore, the system must be able to differentiate between expressions of doubt and objections; since we are assuming that people are engaged in a cooperative dialogue and communicate beliefs that they intend to be recognized, if $\mathrm{S} 1$ were certain of both (a) and (c), then S1 would object to (2), not simply express doubt at it. In summary, the surface form of utterances is one way that speakers convey belief. But these surface forms convey more than just belief and disbelief; they convey multiple strengths of belief, the recognition of which is necessary for identifying whether an agent holds the requisite beliefs for some discourse actions.

We maintain a belief model for each participant which captures these multiple strengths of belief. We contend that at least three strengths of belief must be represented: certain belief ( $a$ belief strength of $\mathrm{C}$ ); strong but uncertain belief, as in (3) above (a belief strength of S); and a weak belief, as in I think that Dr. C might be an education instructor (a belief strength of W). Therefore, our model maintains three degrees of belief, three degrees of disbelief (indicated by attaching a subscript of $N$, such as $S_{N}$ to represent strong disbelief and $W_{N}$ to represent weak disbelief), and one degree indicating no belief about a proposition (a belief strength of 0 ). ${ }^{5}$ Our belief model uses belief intervals to specify the range of strengths

\footnotetext{
${ }^{5}$ Others (Walker, 1991; Galliers, 1991) have also argued for multiple strengths of belief, basing the strength of belief on the amount and kind of evidence available for that belief. We have not investigated how much evidence is needed for an agent to have a particular amount of confidence in a belief; our work has concentrated on recognizing how the strength of belief is communicated in a discourse and the impact that the different belief strengths have on the recognition of discourse acts.
} 
within which an agent's beliefs are thought to fall, and our discourse recipes use belief intervals to specify the range of strengths that an agent's beliefs may assume. Intervals such as $\left[b_{i}: b_{j}\right]$ specify a strength of belief within $b_{i}$ and $b_{j}$, inclusive. For example, the goal of the Inform recipe in Figure 1, (believe( agent2, -prop, [C:C])), is that agent 1 be certain that _prop is true; on the other hand, believe(agent1, -prop, [W:C]), means that _agent 1 must have some belief in _prop.

In order to recognize other beliefs, such as (b) and (c), it is necessary to use more information than just a speaker's utterances. For example, S2 might attribute (c) to S1 because S2 believes that most people think that only one professor teaches each course. Our system incorporates these commonly held beliefs by maintaining a model of a stereotypical user whose beliefs may be attributed to the user during the conversation as appropriate. People also communicate their beliefs by their acceptance (explicit and implicit) and non-acceptance of other people's actions. Thus, explicit or implicit acceptance of discourse actions provides another mechanism for updating the belief model: when an action is recognized as successful, we update our model of the user's beliefs with the effects and goals of the completed action. For example, in determining whether (3) is expressing doubt at (2), thereby implicitly indicating that (2) has been understood and that the Tell action has therefore been successful, the system tentatively hypothesizes that the effects and goals of the Tell action hold, the goal being that $\mathrm{S} 1$ believes that $\mathrm{S} 2$ believes that $\mathrm{Dr}$. Smith is teaching Architecture (belief (b) above). If the system determines that this Express-Doubt inference is the most coherent interpretation of (3), it attributes the hypothesized beliefs to S1. So, our model captures many of the ways in which people infer beliefs: 1) from the surface form of utterances; 2) from stereotype models; and 3) from acceptance (explicit or implicit) or non-acceptance of previous actions.

\section{Combining Knowledge Sources}

Grosz and Sidner (1986) contend that modeling discourse requires integrating different kinds of knowledge in a unified framework in order to constrain the possible role that an utterance might be serving. We use three kinds of knowledge, 1) contextual information provided by previous utterances; 2) world knowledge; and 3) the linguistic information contained in each utterance. Contextual knowledge in our model is captured by the DM and the current focus of attention within it. The system's world knowledge contains facts about the world, the system's beliefs (including its beliefs about a stereotypical user's beliefs), and knowledge about how to go about performing discourse, problem-solving, and domain actions. The linguistic knowledge that we exploit includes the surface form of the utterance, which conveys beliefs and the strength of belief, as discussed in the preceding section, and linguistic clue words. Certain words often suggest what type of discourse action the speaker might be pursuing (Litman and Allen, 1987; Hinkelman, 1989). For example, the linguistic clue please suggests a request discourse act (Hinkelman, 1989) while the clue word but suggests a non-acceptance discourse act. Our model takes these linguistic clues into consideration in identifying the discourse acts performed by an utterance.

Our investigation of naturally occurring dialogues indicates that listeners use a combination of information to determine what a speaker is trying to do in saying something. For example, S2's world knowledge of commonly held beliefs enabled S2 to determine that S1 probably believes (c), and therefore infer that $\mathrm{S} 1$ was expressing doubt at (2). However, S1 might have said (4) instead of (3).

(4) But didn't Dr. Smith win a teaching award? It is not likely that $\mathrm{S} 2$ would think that people typically believe that Dr. Smith winning a teaching award implies that she is not teaching Architecture. However, S2 would probably still recognize (4) as an expression of doubt because the linguistic clue but suggests that (4) may be some sort of non-acceptance action, there is nothing to suggest that S1 does not believe that Dr. Smith winning a teaching award implies that she is not teaching Architecture, and no other interpretation seems more coherent. Since linguistic knowledge is present, less evidence is needed from world knowledge to recognize the discourse actions being performed (Grosz and Sidner, 1986).

In our model, if a new utterance contributes to a discourse action already in the DM, then there must be an inference path from the utterance that links the utterance up to the current tree structure on the discourse level. This inference path will contain an action that determines the relationship of the utterance to the DM by introducing new parameters for which there are many possible instantiations, but which must be instantiated based on values from the DM in order for the path to terminate with an action already in the DM. We will refer to such actions as e-actions since we contend that there must be evidence to support the inference of these actions. By substituting values from the DM that are not present in the semantic representation of the utterance for the new parameters in e-actions, we are hypothesizing a relationship between the new utterance and the existing discourse level of the DM.

Express-Doubt is an example of an e-action (Figure 1). From the speaker's conveying uncertain belief in the proposition _prop2, plan chaining suggests that the speaker might be expressing doubt at some proposition -prop1, and from this Express-Doubt action, further plan chaining may suggest a sequence of actions terminating at an Inform action already in the DM. The ability of -prop1 to unify with the proposition that was conveyed by the Inform action (and rule to unify 
with a rule in the system's world knowledge) is not sufficient to justify inferring that the current utterance contributes to an Express-Doubt action which contributes to an Inform action; more evidence is needed. This is further discussed in Lambert and Carberry (1992).

Thus we need evidence for including eactions on an inference path. The required evidence for e-actions may be provided by linguistic knowledge that suggests certain discourse actions (e.g., the evidence that (4) is expressing doubt) or may be provided by world knowledge that indicates that the applicability conditions for a particular action hold (e.g., the evidence that (3) is expressing doubt).

Our model combines these different knowledge sources in our plan recognition algorithm. From the semantic representation of an utterance, higher level actions are inferred using plan inference rules (Allen, 1979). If the applicability conditions for an inferred action are not plausible, this action is rejected. If the applicability conditions are plausible, then the beliefs contained in them are temporarily ascribed to the user (if an inference line containing this action is later adopted as the correct interpretation, these applicability conditions are added to the belief model of the user). The focus of attention and focusing heuristics (discussed in Lambert and Carberry (1991)) order these sequences of inferred actions, or inference lines, in terms of coherence. For those inference lines with an e-action, linguistic clues are checked to determine if the action is suggested by linguistic knowledge, and world knowledge is checked to determine if there is evidence that the applicability conditions for the e-action hold. If there is world and linguistic evidence for the e-action of one or more inference lines, the inference line that is closest to the focus of attention (i.e., the most contextually coherent) is chosen. Otherwise, if there is world or linguistic evidence for the e-action of one or more inference lines, again the inference line that is closest to the focus of attention is chosen. Otherwise, there is no evidence for the e-action in any inference line, so the inference line that is closest to the current focus of attention and contains no e-action is chosen.

\section{Example}

The following example, an expansion of utterances (1), (2), and (3) from Section 3, illustrates how our model handles 1) implicit and explicit acceptance; 2) negotiation subdialogues embedded within other negotiation subdialogues; 3) expressions of doubt at both immediately preceding and earlier utterances; and 4) multiple expressions of doubt at the same proposition. We will concentrate on how S1's utterances are understood and assimilated into the DM.
(5) S1: What is Dr. Smith teaching?

(6) S2: Dr. Smith is teaching Architecture.

(7) S1: Isn't Dr. Brown teaching Architecture?

(8) S2: No.

(9) Dr. Brown is on sabbatical.

(10) S1: But didn't I see him on campus yesterday?

(11) S2: Yes.

(12) He was giving a University colloguium.

(13) S1: $O K$.

(14) But isn't Dr. Smith a theory person?

The inferencing for utterances similar to (5) and (6) is discussed in depth in Lambert and Carberry (1992), and the resultant DM is given in Figure 2. No clarification or justification of the Request action or of the content of the question has been addressed by either S1 or S2, and S2 has provided a relevant answer, so both parties have implicitly indicated (Clark and Schaefer, 1989) that they think that $S 1$ has made a reasonable and understandable request in asking the question in (5).

The surface form of (7) suggests that $\mathrm{S} 1$ thinks that Dr. Brown is teaching Architecture, but isn't certain of it. This belief is entered into the system's model of S1's beliefs. This surface question is one way to Convey-UncertainBelief. As discussed in Section 3, the most coherent interpretation of (7) based on focusing heuristics, addressing the understandability of (6), is rejected (because there is not evidence to support this inference), so the system tries to relate (7) to the Inform action in (6); that is, the system tries to interpret (7) as addressing the believability of (6). Plan chaining determines that the Convey-Uncertain-Belief action could be part of an Express-Doubt action which could be part of an Address-Unacceptance action which could be an action in an Address-Believability discourse action which could in turn be an action in the Inform action of (6). Express-Doubt is an e-action because the action header introduces new arguments that have not appeared previously on the inference path (see Figure 1). Since there is evidence from world knowledge that the applicability conditions hold for interpreting (7) as an expression of doubt and since there is no other evidence for any other e-action, the system infers that this is the correct interpretation and stops. Thus, (7) is interpreted as an Express-Doubt action. S2's response in (8) and (9) indicates that S2 is trying to resolve $\mathrm{S} 1$ and $\mathrm{S} 2$ 's conflicting beliefs. The structure that the DM has built after these utterances is contained in Figure $3,{ }^{6}$ above the numbers (5) (9).

The Surface-Neg-YN-Question in utterance (10) is one way to Convey-Uncertain-Belief. The linguistic clue but suggests that $\mathrm{S} 1$ is execut-

\footnotetext{
${ }^{6}$ For space reasons, only inferencing of discourse actions will be discussed here, and only action names on the discourse level are shown; the problem-solving and domain levels are as shown in Figure 2.
} 


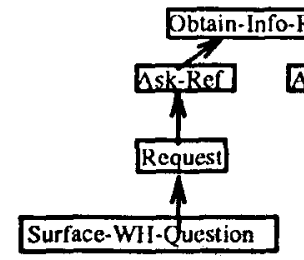

(5)

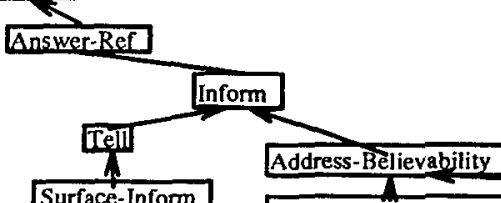

(6)

Address-Unacceptance

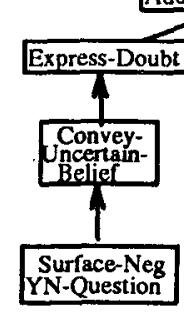

(7)

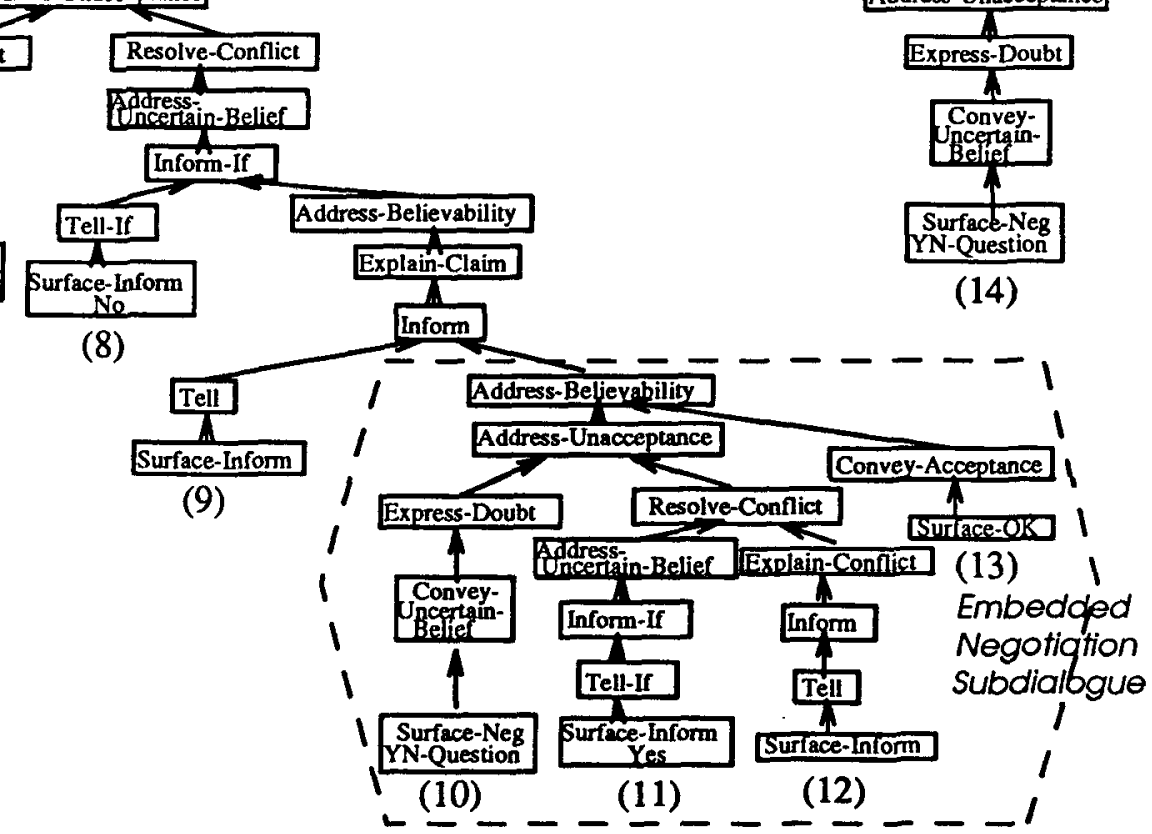

Address-Unacceptance contan

urface-Ne

Figure 3. Discourse Level of DM for Dialogue in Section 6

ing a non-acceptance discourse action; this nonacceptance action might be addressing either (9) or $(6)$. Focusing heuristics suggest that the most likely candidate is the Inform act attempted in (9), and plan chaining suggests that the ConveyUncertain-Belief could be part of an ExpressDoubt action which in turn could be part of an Address-Unacceptance action which could be part of an Address-Believability action which could be part of the Inform action in (9). Again, there is evidence that the applicability conditions for the e-action (the Express-Doubt action) hold: world knowledge indicates that a typical user believes that professors who are on sabbatical are not on campus. Thus, there is both linguistic and world knowledge giving evidence for the Express-Doubt action (and no other e-action has both linguistic and world knowledge evidence), so (10) is interpreted as expressing doubt at (9).

In (11) and (12), S2 clears up the confusion that $\mathrm{S} 1$ has expressed in (10), by telling S1 that the rule that people on sabbatical are not on campus does not hold in this case. In (13), S1 indicates explicit acceptance of the previously communicated proposition, so the system is able to determine that $\mathrm{S} 1$ has accepted S2's response in (12). This additional negotiation, utterances (10)(13), illustrates our model's handling of negotiation subdialogues embedded within other negotiation subdialogues. The subtree contained within the dashed lines in Figure 3 shows the structure of this embedded negotiation subdialogue.

The linguistic clue but in (14) then again suggests non-acceptance. Since (12) has been explicitly accepted, (14) could be expressing nonacceptance of the information conveyed in either (9) or (6). Focusing heuristics suggest that (14) is most likely expressing doubt at (9). World knowledge, however, provides no evidence that the applicability conditions hold for (14) expressing doubt at (9). Thus, there is evidence from linguistic knowledge for this inference, but not from world knowledge. The system's stereotype model does indicate, however, that it is typically believed that faculty only teach courses in their field and that Architecture and Theory are different fields. So in this case, the system's world knowledge provides evidence that Dr. Smith being a theory person is an indication that Dr. Smith does not teach Architecture. Therefore, the system interprets (14) as again expressing doubt at (6) because there is evidence for this inference from both world and linguistic knowledge. The system infers therefore that $\mathrm{S} 1$ has implicitly accepted the statement in (9), that Dr. Smith is on sabbatical. Thus, the system is able to recognize and assimilate a second expression of doubt at the proposition conveyed in (6). The DM for the discourse level of the entire dialogue is given in Figure 3. 


\section{Conclusion}

We have presented a plan-based model that handles cooperative negotiation subdialogues by inferring both the communicative actions that people pursue when speaking and the beliefs underlying these actions. Beliefs, and the strength of those beliefs, are recognized from the surface form of utterances and from the explicit and implicit acceptance of previous utterances. Our model combines linguistic, contextual, and world knowledge in a unified framework that enables recognition not only of when an agent is negotiating a conflict between the agent's beliefs and the preceding dialogue but also which part of the dialogue the agent's beliefs conflict with. Since negotiation is an integral part of multi-agent activity, our model addresses an important aspect of cooperative interaction and communication.

\section{References}

Allen, James F. (1979). A Plan-Based Approach to Speech Act Recognition. PhD thesis, University of Toronto, Toronto, Ontario, Canada.

Carberry, Sandra (1987). Pragmatic Modeling: Toward a Robust Natural Language Interface. Computational Intelligence, 3, 117-136.

Clark, Herbert and Schaefer, Edward (1989). Contributing to Discourse. Cognitive Science, 259-294.

Cohen, Robin (1987). Analyzing the Structure of Argumentative Discourse. Computational Linguistics, 13(1-2), 11-24.

Cohen, Robin and Young, Mark A. (1991). Determining Intended Evidence Relations in Natural Language Arguments. Computational Intelligence, $7,110-118$.

Flowers, Margot, McGuire, Rod, and Birnbaum, Lawrence (1982). Adversary Arguments and the Logic of Personal Attack. In W. Lehnert and M. Ringle (Eds.), Strategies for Natural Language Processing (pp. 275-294). Hillsdage, New Jersey: Lawrence Erlbaum Assoc.

Galliers, Julia R. (1991). Belief Revision and a Theory of Communication. Technical Report 193, University of Cambridge, Cambridge, England.

Goodman, Bradley A. and Litman, Diane J. (1992). On the Interaction between Plan Recognition and Intelligent Interfaces. User Modeling and User-Adapted Interaction, 2, 83-115.

Grosz, Barbara and Sidner, Candace (1986). Attention, Intention, and the Structure of Discourse. Computational Linguistics, 12(3), $175-204$
Hinkelman, Elizabeth (1989). Two Constraints on Speech Act Ambiguity. In Proceedings of the 27th Annual Meeting of the ACL (pp. 212219), Vancouver, Canada.

Kautz, Henry and Allen, James (1986). Generalized Plan Recognition. In Proceedings of the Fifth National Conference on Artificial Intelligence (pp. 32-37), Philadelphia, Pennsylvania.

Lambert, Lynn and Carberry, Sandra (1991). A Tripartite Plan-based Model of Dialogue. In Proceedings of the 29th Annual Meeting of the $A C L$ (pp. 47-54), Berkeley, CA.

Lambert, Lynn and Carberry, Sandra (1992). Using Linguistic, World, and Contextual Knowledge in a Plan Recognition Model of Dialogue. In Proceedings of COLING-92, Nantes, France. To appear.

Litman, Diane and Allen, James (1987). A Plan Recognition Model for Subdialogues in Conversation. Cognitive Science, 11,163-200.

Perrault, Raymond (1990). An Application of Default Logic to Speech Act Theory. In P. Cohen, J. Morgan, and M. Pollack (Eds.), Intentions in Communication (pp. 161-185). Cambridge, Massachusetts: MIT Press.

Pollack, Martha (1990). Plans as Complex Mental Attitudes. In P. R. Cohen, J. Morgan, and M. E. Pollack (Eds.), Intentions in Communication (pp 77-104). MIT Press.

Quilici, Alexander (1991). The Correction Machine: A Computer Model of Recognizing and Producing Belief Justifications in Argumentative Dialogs. PhD thesis, Department of Computer Science, University of California at Los Angeles, Los Angeles, California.

Ramshaw, Lance A. (1991). A Three-Level Model for Plan Exploration. In Proceedings of the 29th Annual Meeting of the ACL (pp. 36-46), Berkeley, California.

Reichman, Rachel (1981). Modeling Informal Debates. In Proceedings of the 1981 International Joint Conference on Artificial Intelligence (pp. 19-24), Vancouver, B.C. IJCAI.

Sidner, Candace L. (1985). Plan Parsing for Intended Response Recognition in Discourse. Computational Intelligence, 1, 1-10.

Walker, Marilyn (1991). Redundancy in Collaborative Dialogue. Presented at The $A A A I$ Fall Symposium: Discourse Structure in Natural Language Understanding and Generation (pp. 124-129), Asilomar, CA.

Wilensky, Robert (1981). Meta-Planning: Representing and Using Knowledge About Planning in Problem Solving and Natural Language Understanding. Cognitive Science, 5, 197-233. 